\title{
Stratigraphic analysis of intercalated graphite electrodes in aqueous inorganic acid solutions
}

\author{
Stefania De Rosa ${ }^{1,2}$, Paolo Branchini ${ }^{1}$, Valentina Spampinato ${ }^{3}$, Alexis Franquet ${ }^{3}$, Rossella Yivlialin ${ }^{4}$, Lamberto \\ Duò $^{5}$, Gianlorenzo Bussetti ${ }^{5}$, and Luca Tortora ${ }^{1,6}(\bowtie)$ \\ ${ }^{1}$ LASR3 Surface Analysis Laboratory, INFN Roma Tre, via della Vasca Navale 84, 00146 Rome, Italy \\ ${ }^{2}$ Department of Mathematics and Physics, Roma Tre University, via della Vasca Navale 84, 00146 Rome, Italy \\ ${ }^{3}$ Imec, Kapeldreef 75, B-3001 Leuven, Belgium \\ ${ }^{4}$ Helmholtz-Zentrum Berlin für Materialen und Energie GmbH, Institute for Solar Fuels (EE-IF), Hahn-Meitner-Platz 1, D-14109 Berlin, \\ Germany \\ ${ }^{5}$ Department of Physics, Politecnico di Milano, Piazza Leonardo da Vinci 32, I-20133 Milano, Italy \\ ${ }^{6}$ Department of Science, Roma Tre University, via della Vasca Navale 84, 00146 Rome, Italy
}

(C) The Author(s) 2021

Received: 24 March 2021 / Revised: 11 May 2021 / Accepted: 20 May 2021

\begin{abstract}
A detailed stratigraphic investigation of the intercalation mechanism when graphite electrodes are immersed inside diluted perchloric $\left(\mathrm{HClO}_{4}\right)$ and sulfuric $\left(\mathrm{H}_{2} \mathrm{SO}_{4}\right)$ electrolytes is obtained by comparing results when graphite crystals are simply immersed in the same acid solutions. By combining time-of-flight secondary ion mass spectrometry (ToF-SIMS) and in-situ atomic force microscopy (AFM), we provide a picture of the chemical species involved in the intercalation reaction. The depth intensity profile of the ion signals along the electrode crystal clearly shows a more complex mechanism for the intercalation process, where the local morphology of the basal plane plays a crucial role. Solvated anions are mostly located within the first tens of nanometers of graphite, but electrolytes also diffuse inside the buried layers for hundreds of nanometers, the latter process is also aided by the presence of mesoscopic crystal defects. Residual material from the electrolyte solution was found localized in well-defined circular spots, which represent preferential interaction areas. Interestingly, blister-like micro-structures similar to those observed on the highly oriented pyrolytic graphite (HOPG) surface were found in the buried layers, confirming the equivalence of the chemical condition on the graphite surface and in the underneath layers.
\end{abstract}

\section{KEYWORDS}

highly oriented pyrolytic graphite (HOPG), intercalation, graphite, graphene, time-of-flight secondary ion mass spectrometry (ToF-SIMS), atomic force microscopy (AFM)

\section{Introduction}

Ion batteries development (by exploiting the change of the transport properties) and, more recently, graphene industrial production (by quenching the layer-layer interaction) are two important examples of technological areas where anion intercalation into stratified crystal structures plays a crucial role [1-3]. The stratified crystals prototype, namely highly oriented pyrolytic graphite (HOPG), consisting of honeycomb graphene layers stacked parallel with each other. The graphene exfoliation can be achieved by a wide range of chemical procedures in liquid-phase [4-7]. One of the more tunable and advantageous methods is based on electrochemistry $[8,9]$. Historically, perchloric $\left(\mathrm{HClO}_{4}\right)$ and sulfuric $\left(\mathrm{H}_{2} \mathrm{SO}_{4}\right)$ acids represent suitable electrolytes in mild aqueous solutions for graphite treatment and oxidation [10-12]. By applying proper electrochemical (EC) potentials, solvated $\mathrm{ClO}_{4}^{-}$and $\mathrm{HSO}_{4}^{-}$ ions percolate between the HOPG layers. HOPG thus evolves into the so-called graphite intercalated compound (GIC), which shows different transport properties with respect to the pristine sample [13-15]. In addition, the presence of ions within the interlayer spacing of graphite helps its delamination in liquid-phase [16]. GIC is considered a new kind of crystal having the intercalated ions in between the ordered arrays of one or more graphite layers (see below for details) [17-19]. The most relevant morphological change on the crystal surface after the graphite intercalation is the formation of blisters [17, 18]. The latter, bubble-like circular structures (with lateral sizes within hundreds of $\mathrm{nm}$ and up to the micrometer length scale; height above tens of $\mathrm{nm}$ ) which swell the HOPG basal plane, are generally interpreted as a consequence of the evolution of gaseous molecular species $\left(\mathrm{CO}, \mathrm{CO}_{2}\right.$, and $\left.\mathrm{O}_{2}\right)$ during the oxidative process [20-23]. The presence of such gases was recently detected by a mass-spectrometer just placed above the electrode surface [24]. If blisters induce a detriment of the HOPG surface, the gas production reasonably also affects the crystal bulk (by the formation of defects, dislocations, strains, etc.). Because of this, having a complete picture of the HOPG surface and bulk chemical evolution during the exfoliation process becomes crucial. The local morphological changes occurring on the HOPG basal plane have been extensively studied by microscopic techniques, like electrochemical-atomic 
force microscopy (EC-AFM) [12, 25]. More recently, it has been possible to monitor the appearance of blisters by combining electrochemical-scanning tunneling microscopy (EC-STM), cyclic voltammetry (CV), and normal-pulse voltammetry (NPV) [26-29]. All results from these works demonstrate an interdependence between the HOPG surface swelling and the reaching of the oxygen evolution potential (OEP). These new results have refined the current interpretative model used for the morphological characterization of the electrode [18]. A similar experimental effort is now wished for a more local chemical analysis of the intercalation process. To this goal, Raman spectroscopy has been initially exploited allowing an in-situ characterization during the EC reaction [23]. However, in a very recent work, we employed time-of-flight secondary ion mass spectrometry (ToF-SIMS) technique proving that a more sensitive analysis of the chemical species present on the HOPG electrode surface after the first intercalation stage is possible despite only ex-situ measures are possible [30]. By using this technique, the correlation between chemical and morphological changes can be highlighted by combining mass spectra and chemical maps $[31,32]$. In this work, in order to investigate the intercalation also in the sub-surface regions of the crystals, we exploited the so-called dual beam ToF-SIMS depth profiling experiments at very low sputter energy. The use of low energy $\mathrm{Cs}^{+}$ions as a sputtering source has been already demonstrated to be efficient for characterizing hybrid organic/inorganic architectures with a sub-nanometer depth resolution [33,34]. Besides, thanks to an innovative experimental set-up, which combines ToF-SIMS and in-situ atomic force microscopy (AFM) measures, we were able to monitor and spatially localize the penetration depth of the molecular species involved in the intercalation process. Molecular species coming from the electrolyte $\left(\mathrm{ClO}_{4}^{-}\right.$and $\left.\mathrm{SO}_{4} \mathrm{H}^{-}\right)$, as well as oxygenbased molecular fragment ions generated as a consequence of the EC treatment $\left(\mathrm{CO}_{2} \mathrm{H}^{-}\right.$and $\left.\mathrm{O}_{2}^{-}\right)$, were revealed and located within the graphitic planes.

\section{Experimental}

\subsection{Sample preparation}

Sulfuric acid (99.999\%, Sigma-Aldrich) and perchloric acid (70\%, ACS reagent, Sigma-Aldrich) were utilized in the diluted (2 M) $\mathrm{HClO}_{4}$ and $(1 \mathrm{M}) \mathrm{H}_{2} \mathrm{SO}_{4}$ acid solutions for treating the samples. The solutions were purified by bubbling pure Ar in a separator funnel for several hours. The different concentrations for the two acids allow the presence of a comparable number of anions in solution. Pristine z-grade HOPG samples (by Optigraph) were electrochemically intercalated or only immersed [for some minutes in view of having comparable time intervals to those ones required for performing the cyclic-voltammetries (CV), see below] in the two acid solutions. The simple crystal immersion was used as a countercheck for the final data interpretation. In fact, the ex-situ ToF-SIMS measures oblige the graphite extraction from the acid solution bath and the consequent risk of having residuals and precipitates caused by the sample drying. For the electrochemical treatment, HOPG crystal was used as a working electrode (WE) in a threeelectrode EC cell, where two Pt wires were used for the counter electrode (CE) and the quasi-reference electrode (PtQRef). The latter is not a proper electrode based on a redox couple, but it shows a stable (within few millivolts) shift of $+0.743 \mathrm{~V}$ versus the standard hydrogen electrode (SHE) immersed in the same electrolyte. CV (scan rate of $25 \mathrm{mV} / \mathrm{s}$ ) was performed from 0.3 to $1.1 \mathrm{~V}$ (in $\mathrm{HClO}_{4}$ ) and from 0.3 to $1.3 \mathrm{~V}$ (in $\mathrm{H}_{2} \mathrm{SO}_{4}$ ) to reach the IV intercalation stage in both the electrolytes.
After the EC process, the HOPG samples were dried under gentle nitrogen flux.

\subsection{ToF-SIMS/in-situ AFM analysis}

HOPG crystals were analyzed with a ToF-SIMS/AFM combined tool (ToF-SIMS NCS, IONTOF GmbH, Münster, Germany). Such an instrument allows the suspension of the ToF-SIMS depth profile experiment and a subsequent transfer of the sample stage to the AFM position without breaking the vacuum (see Fig. S1 in the Electronic Supplementary Material (ESM)). During the sample stage transfer, the submicron positioning accuracy is guaranteed by the stage calibration. Regarding the dual beam ToF-SIMS depth profile conditions, $\mathrm{Bi}_{1}{ }^{+}$at $30 \mathrm{keV}$ in bunched mode was used as a primary beam and low energy $\mathrm{Cs}^{+}$ion source $(250 \mathrm{eV})$ as an erosion gun. The analysis raster of $100 \mu \mathrm{m} \times 100 \mu \mathrm{m}$ was set at the center of the sputtered area of $300 \mu \mathrm{m} \times 300 \mu \mathrm{m}$. The depth crater values were experimentally established through in-situ AFM surface profile measurements, acquired on the analyzed area before and after each depthprofiling measurement. The values were afterward used for intensity-time to intensity-depth calibration, assuming a constant sputter yield during the depth profiling experiments. The sputter yield value, resulting from the calibration, is consistent with the literature data regarding our experimental conditions [35]. Multiple AFM morphological images and ToF-SIMS chemical maps were acquired on the same areas with the purpose of comparing the topography and the chemical information. AFM was operated in contact mode for all the reported measurements.

\section{Results and discussion}

\subsection{General remarks of the intercalation process}

A schematic representation of the current HOPG intercalation process is reported in Fig. 1. This reaction can be conceived as a two-step process. The first one involves the HOPG basal plane: solvated anions (water molecules are not depicted in Fig. 1) travel through the structural crystal defects reaching also buried graphene layers. The second process stage foresees the anions diffusion within the first graphite layers. At the end of these two steps, anions should be located, along the $z$-direction, in mean-ordered spatial intervals of $n$ graphene
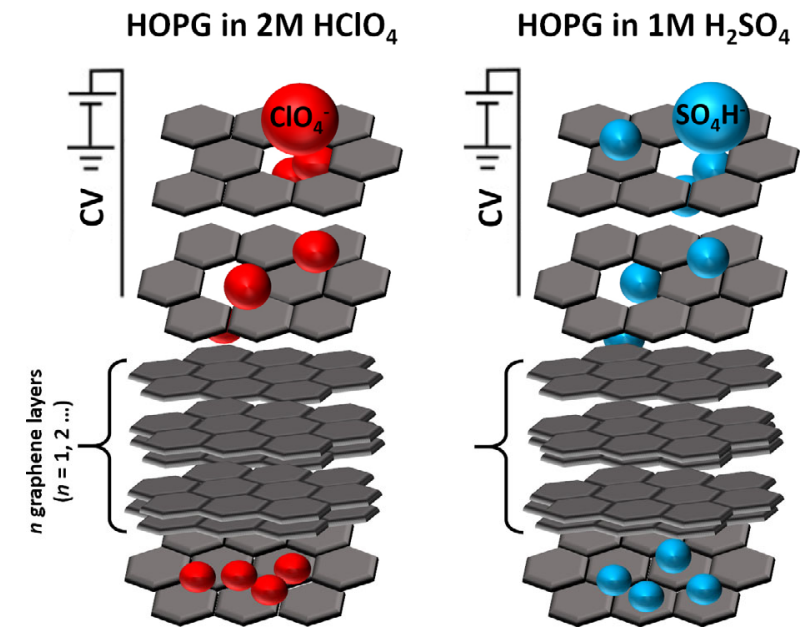

Figure 1 Schematic illustration of the current intercalation model operated on $z$-grade $\mathrm{HOPG}$ samples with acid solutions of $\mathrm{HClO}_{4}$ (left) and, on the right side, $\mathrm{H}_{2} \mathrm{SO}_{4}$ (for more simplicity, anions are not solvated in the picture). The scheme shows the proposed model for the ion intercalation, where the electrolyte anions take advantage from the crystal surface defects to reach the HOPG subsurface. 
layers $(n=1,2 \ldots)$. Up to now, a detailed analysis of this second phase and the effects of the different employed electrolytes have not been fully explored. In Fig. 2, the CVs show the evolution of the Faradaic current through the $\mathrm{HOPG}$ in $\mathrm{HClO}_{4}$ (a) and $\mathrm{H}_{2} \mathrm{SO}_{4}$ (b) electrolytes. The shoulder related to the fourth anion intercalation stage $(n=4)$ is labeled in the positive scans of the voltammograms in Figs. 2(a) and 2(b). The negative peak is traditionally interpreted in terms of a partial ions deintercalation, which gives a quasi-reversible character at the overall reaction [36]. When the fourth intercalation stage is reached during a cyclic-voltammetry, the graphite electrode becomes a so-called GIC. GIC can be considered a new crystal where an ion layer is interposed in the original stratified crystal structure after $n=4$ graphite layers. Our chemical and morphological investigation is focused only on the IV intercalation stage because it ensures that the main processes and reactions occur without a massive detriment of the electrode crystal.

\subsection{Chemical and morphological analysis of the real surface and buried graphite layers}

Pristine HOPG sample was initially studied as a reference sample for the ToF-SIMS investigation (see Fig. S2 in the ESM). After the EC treatments, the HOPG was analyzed by AFM. Typically, the EC treatment produces blisters, carbon dissolution, and a general surface detriment $[26,37]$. The complexity of the morphology increases when the electrode surface is investigated ex-situ. In this case, some residuals from the electrolyte can also affect the quality of the AFM image. In Fig. 3, we report two representative AFM images of the HOPG real surface upon reaching the fourth stage of intercalation in $\mathrm{HClO}_{4}$ in Fig. 3(b) and $\mathrm{H}_{2} \mathrm{SO}_{4}$ in Fig. 3(c) electrolytes. In the former case, it is possible to recognize a general surface detriment and some sub-micrometer features (blisters). Blisters are also
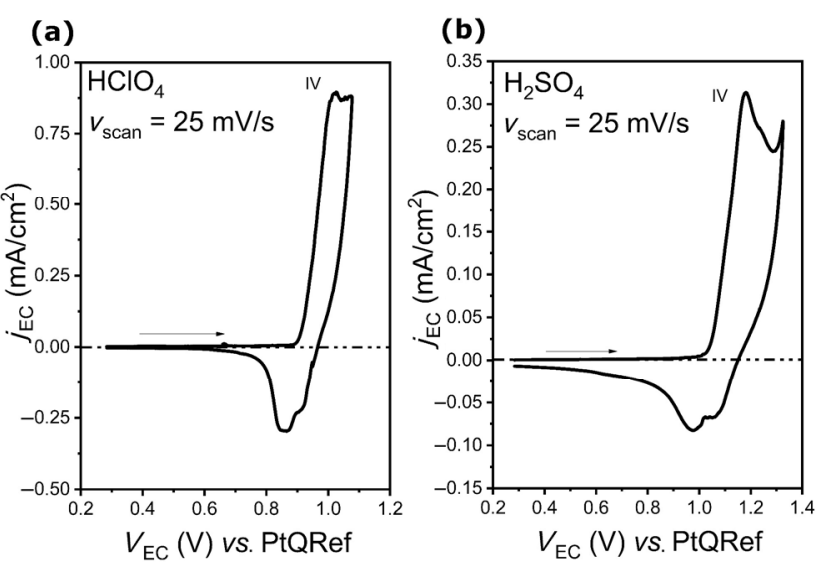

Figure 2 Characteristic CV $\left(v_{\text {scan }}=25 \mathrm{mV} / \mathrm{s}\right)$ acquired on HOPG treated with $2 \mathrm{M} \mathrm{HClO}_{4}$ (a) and $1 \mathrm{M} \mathrm{H}_{2} \mathrm{SO}_{4}$ (b) solution. The reported arrows indicate the $\mathrm{CV}$ scan direction. present in Fig. 3(c), where a larger number of roundish shapes (residuals) are observed. The intercalated samples were thus studied by the ToF-SIMS system. The results are then compared with those acquired on the immersed samples, as previously explained. In ToF-SIMS depth profiles, secondary ion intensities are plotted versus depth. The shaded bar areas of the profile curves represent the ToF-SIMS measurement uncertainty, evaluated on the basis of our experimental conditions. The same uncertainty can be applied to all depth profile results for a better comprehension of the data. In all cases (immersed and intercalated samples) the $\mathrm{C}_{6}{ }^{-}$signal intensity is used as a reference secondary ion for graphite, as already reported in the literature [38]. It reaches a plateau approximatively within the first $4 \mathrm{~nm}$, as clearly visible in Fig. 4 . Intensity signals related to the anions from $\mathrm{HClO}_{4}$ in Fig. 4(a) and $\mathrm{H}_{2} \mathrm{SO}_{4}$ in Fig. 4(b) acids and some oxygen-based ion fragments in Figs. 4(c) and $4(\mathrm{~d})$ are also reported. Considering that the chlorine and sulfur based molecular species are directly involved in the EC process, we would like to figure out possible differences in $\mathrm{ClO}_{4}^{-}$and $\mathrm{SO}_{4} \mathrm{H}^{-}$ions behavior. For what regards the HOPG sample treated with $\mathrm{HClO}_{4}$, it is possible to appreciate that the $\mathrm{ClO}_{4}^{-}$ion (Fig. 4(a)) is more intense in the first $2 \mathrm{~nm}$. The ion is strongly present even in the sample buried layers. This result confirms that the intercalation process proceeds beyond the uppermost graphite layers. On the contrary, in the immersed sample, the $\mathrm{ClO}_{4}^{-}$ion signal intensity rapidly reaches negligible values (blue shaded bar area) at about $4 \mathrm{~nm}$. This result puts in evidence the crucial role of the EC process in forcing the anions inside the HOPG crystal. A similar approach in the evaluation of the EC process was adopted for the sample treated with $\mathrm{H}_{2} \mathrm{SO}_{4}$. In Fig. 4(b) the signal intensity variation of the $\mathrm{SO}_{4} \mathrm{H}^{-}$species is reported. Also in this case, the EC treatment led to a better insertion of the acid molecules and their diffusion throughout the crystal. In the intercalation theoretical model [18], the authors propose that gases (namely $\mathrm{CO}, \mathrm{CO}_{2}$, and $\mathrm{O}_{2}$ ) develop during graphite oxidation. Consequently, we decided to search traces of this process and the electrochemical graphite oxide (EGO) formation in the evolution of $\mathrm{O}_{2}$ and $\mathrm{CO}_{2} \mathrm{H}$ molecular species. In Figs. 4(c) and 4(d), the behavior of $\mathrm{O}_{2}^{-}$ and $\mathrm{CO}_{2} \mathrm{H}^{-}$ion signals for the intercalated sample in perchloric and sulfuric acid, respectively, is reported. The signals show an initial increase of intensity, with a maximum within the first $2 \mathrm{~nm}$ (sample real surface). As already seen for the $\mathrm{ClO}_{4}^{-}$ ion, the signal intensity for both ions remains pretty constant even in the HOPG buried layers. The presence of such ions within the first tens of nanometers confirms the strong oxidative action operated by the acid during the EC treatment with a subsequent formation of related gaseous products. In addition, these findings agree and give a rationale of previous results obtained by Alliata and coworkers [25], where the authors deduce an intercalation depth of about a hundred $\mathrm{nm}$ as a consequence of the swelling of the HOPG basal plane as observed by an in-situ AFM analysis. When the sample is only
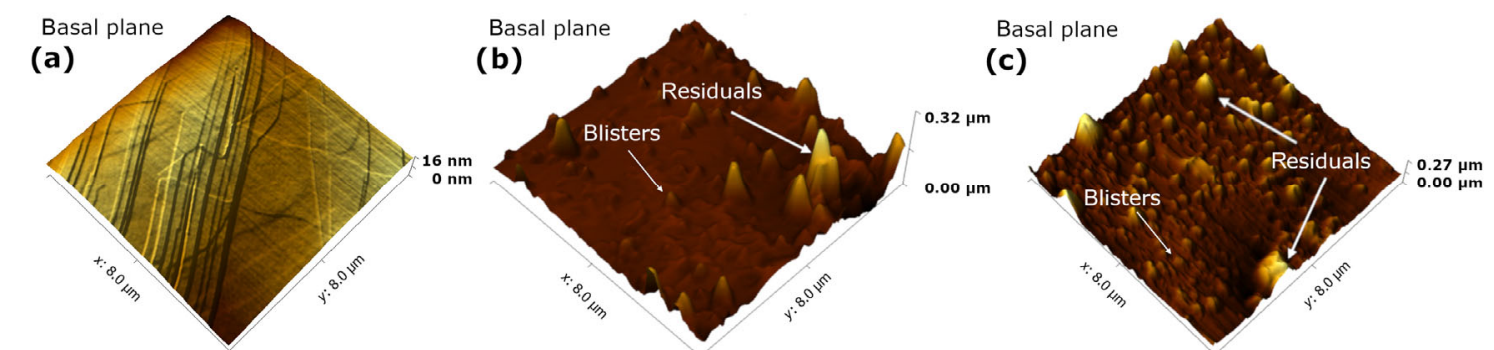

Figure 3 AFM images $(8 \mu \mathrm{m} \times 8 \mu \mathrm{m})$ of HOPG pristine surface (a), HOPG surface intercalated in $2 \mathrm{M} \mathrm{HClO}_{4}$ solution (b), and in $1 \mathrm{M} \mathrm{H}_{2} \mathrm{SO}_{4}$ solution (c). For all images, three dimensional (3D) AFM topography are shown. 

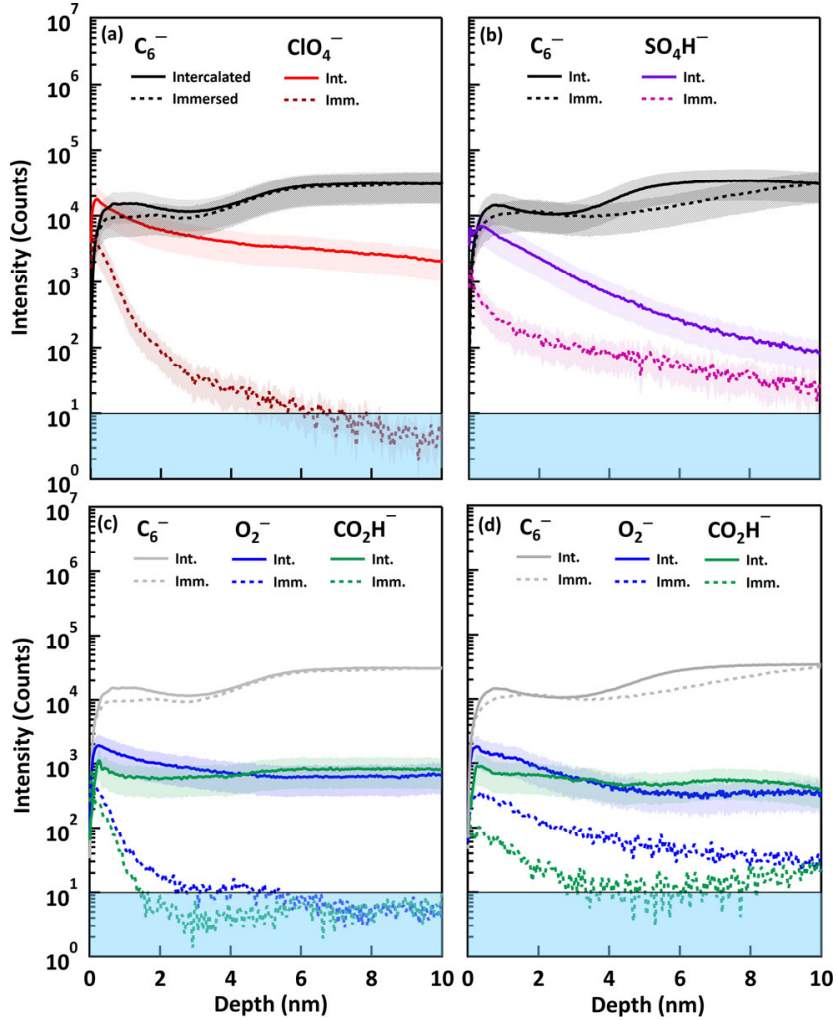

Figure 4 ToF-SIMS depth profiles of HOPG intercalated and immersed in $\mathrm{HClO}_{4}$ solution (a) and (c) and in $\mathrm{H}_{2} \mathrm{SO}_{4}$ solution (b) and (d). Secondary ion intensities of $\mathrm{C}_{6}^{-}$at $m / z 72$, and $\mathrm{ClO}_{4}{ }^{-}$at $\mathrm{m} / z$ 98.94 are shown in (a) for immersed and intercalated samples in $\mathrm{HClO}_{4}$ solution; $\mathrm{C}_{6}^{-}$, and $\mathrm{SO}_{4} \mathrm{H}^{-}$ at $m / z 96.95$ are shown in (b) for immersed and intercalated samples in $\mathrm{H}_{2} \mathrm{SO}_{4}$ solution; $\mathrm{O}_{2}^{-}$at $m / z 31.99$, and $\mathrm{CO}_{2} \mathrm{H}^{-}$at $\mathrm{m} / z 44.99$ are shown in (c) for immersed and intercalated samples in $\mathrm{HClO}_{4}$ solution and in (d) for immersed and intercalated samples in $\mathrm{H}_{2} \mathrm{SO}_{4}$ solution. All secondary ion intensities are plotted versus depth, starting from the surface to $10 \mathrm{~nm}$. The shaded bar areas of the profile curves represent the ToF-SIMS measurement uncertainty, evaluated on the basis of our experimental conditions.

immersed in the acid, the $\mathrm{O}_{2}^{-}$and $\mathrm{CO}_{2} \mathrm{H}^{-}$signal behavior starts with a fairly intense value at the surface and it rapidly decreases in intensity. These results shed a light on the importance of studying the effects of the EC treatment on the HOPG buried layers.

While the correlation between the surface chemistry and the microscopic analysis has been already discussed [30], a similar investigation is still lacking for the buried graphite layers. Therefore, we have broadened our interests considering a wide depth range (stratigraphic analysis from 0 to $165 \mathrm{~nm}$ ) and by combing microscopic and chemical analysis using the ToF-SIMS/AFM set-up. In Fig. 5, results from depth profiling experiments on a sample intercalated with $\mathrm{HClO}_{4}$ are presented. $\mathrm{C}_{6}^{-}, \mathrm{ClO}_{4}^{-}, \mathrm{O}_{2}^{-}$, and $\mathrm{CO}_{2} \mathrm{H}^{-}$depth profiles (Fig. 5(a)) and chemical maps for $\mathrm{ClO}_{4}{ }^{-}$at specific depth values (basal plane, $4,50,110,140$, and $165 \mathrm{~nm}$ ) are shown. Excluding the $\mathrm{C}_{6}$ signal, all the other ion signals taken into account decrease in intensity as a function of the sample depth (Fig. 5(a)). The ToF-SIMS chemical maps are helpful to rationalize the behavior of the signals. At the basal plane (Fig. 5(b)), the $\mathrm{ClO}_{4}^{-}$twodimensional (2D) distribution reveals the widespread presence of residual acid from the EC treatment. The acid is homogenously distributed till a depth of $4 \mathrm{~nm}$ (limit of the real surface, Fig. 5(c)). In the next tens of nanometers in depth, it is possible to appreciate the exponential decreasing of the $\mathrm{ClO}_{4}^{-}$ion signal intensity (Fig. 5(a)) corresponding to a strong reduction of the number of
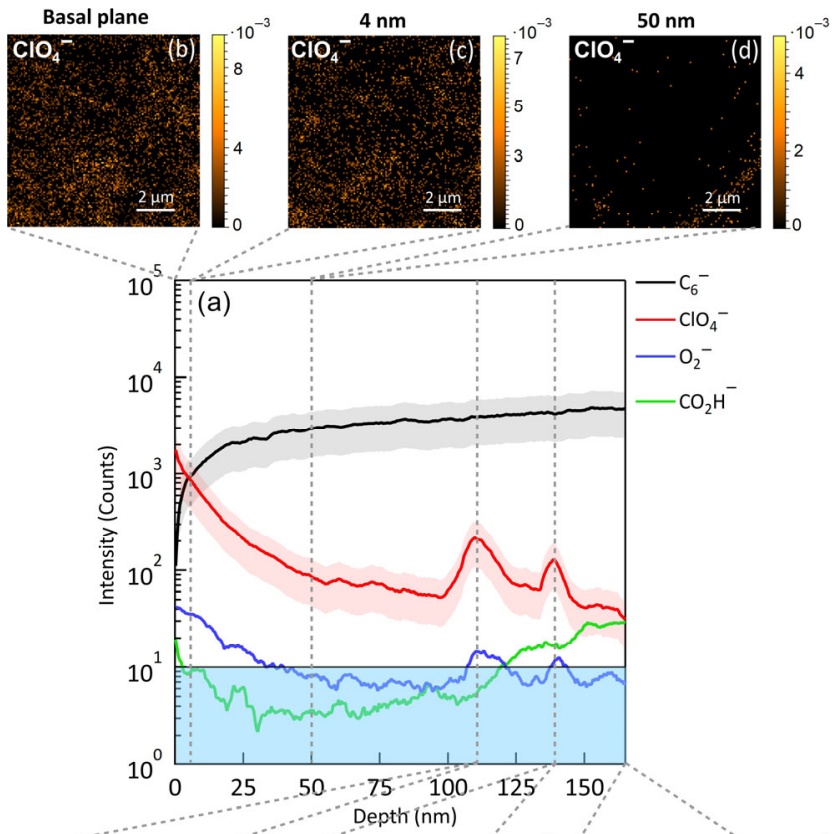

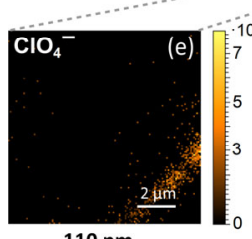

$110 \mathrm{~nm}$

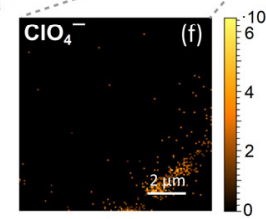

$140 \mathrm{~nm}$

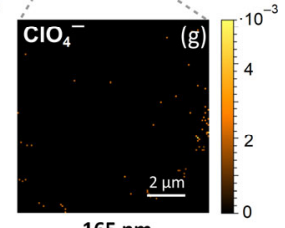

$165 \mathrm{~nm}$
Figure 5 ToF-SIMS depth profiles (a) and images (b)-(g) of HOPG intercalated in $\mathrm{HClO}_{4}$ solution. Secondary ion intensities of $\mathrm{C}_{6}{ }^{-}$at $\mathrm{m} / \mathrm{z} 72$, $\mathrm{ClO}_{4}{ }^{-}$at $\mathrm{m} / \mathrm{z}$ 98.94, $\mathrm{O}_{2}^{-}$at $\mathrm{m} / \mathrm{z} 31.99$, and $\mathrm{CO}_{2} \mathrm{H}^{-}$at $\mathrm{m} / \mathrm{z} 44.99$ are plotted versus depth $(0-165 \mathrm{~nm})$ in the central panel. The normalised ToF-SIMS images $(10 \mu \mathrm{m} \times 10 \mu \mathrm{m})$ show the $\mathrm{ClO}_{4}^{-}$secondary ion in-plane $(X Y)$ distribution at different depths ( $z$ values): basal plane (b), $z=4 \mathrm{~nm}$ (c), $z=$ $50 \mathrm{~nm}(\mathrm{~d}), z=110 \mathrm{~nm}(\mathrm{e}), z=140 \mathrm{~nm}(\mathrm{f}), z=165 \mathrm{~nm}(\mathrm{~g})$.

counts in the chemical map (Fig. 5(d)). Then, an unexpected increase of the signal intensity was observed in buried layers $(z=110$ and $140 \mathrm{~nm})$. A quite similar behavior was detected for $\mathrm{O}_{2}^{-}$and $\mathrm{CO}_{2} \mathrm{H}^{-}$. The co-presence in the graphite bulk of the electrolyte and oxygen-based molecular fragment ions suggests that the electrochemical oxidation can occur also far from the crystal subsurface. Here, the $\mathrm{ClO}_{4}^{-}$ion signal is not homogeneously distributed all over the $X Y$-plane, as previously shown in Figs. 5(b) and 5(c), but it exhibits a very specific distribution (Figs. 5(e) and 5(f)). Thereafter, the ion signal drops again at the end of the depth profiling experiment (Fig. 5(g)). Combining ToF-SIMS chemical maps and AFM images it is possible to highlight at the same time changes in the crystal topography and distribution of the chemical species at the surface as well as in the buried layers. Here, such combined approach aims to correlate the presence of electrolyte residuals with characteristic crystal defects to explain the presence of $\mathrm{ClO}_{4}$ at hundreds of nanometers. AFM measurements performed on the same area at the beginning (top) and the end (bottom) of the sputtering process are shown in Fig. 6.

The crystal basal plane (Fig. 6(a)) is characterized by the presence of blisters and acid residuals. The sputtering process simultaneously removed blisters, electrolyte residuals, and graphite planes putting in evidence some crystal structural defects such as steps and rifts (Fig. 6(b)). Surprisingly, the chemical distribution of $\mathrm{ClO}_{4}^{-}$in the buried layers (Fig. 5(d)-5(f)) seems to perfectly overlap with the crystal rift. Furthermore, right next to the rift clear blisters were identified by AFM. This unforeseen scenario opens an important question concerning 

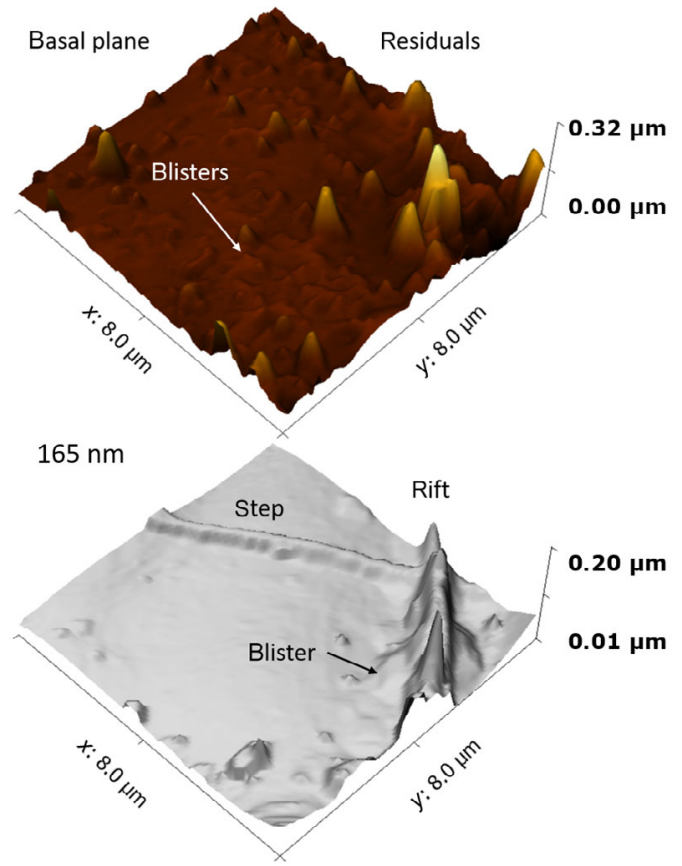

Figure 6 AFM images $(8 \mu \mathrm{m} \times 8 \mu \mathrm{m})$ of $\mathrm{HOPG}$ intercalated in $\mathrm{HClO}_{4}$ solution, acquired on the surface sample before the sputtering process of the ToF-SIMS depth profile measurement (a) and on the same area, after the depth profile measurement (at $z=165 \mathrm{~nm}$ ) (b). For both images, 3D AFM topography is shown.

the role of such defects in the anion intercalation process. In fact, despite being of high quality, HOPG samples have intrinsic defects both on the basal plane and in the crystal bulk. In particular, graphite is constituted by wide grains more or less aligned with the neighbors according to the graphite grade. By using gaps between grains, anions can cover longer paths with respect to those through not-aligned defects present in the different graphite stratified layers. This scenario was also validated for the HOPG samples treated with $\mathrm{H}_{2} \mathrm{SO}_{4}$. In Fig. 7, ToF-SIMS images were reconstructed as $X Y$ slices at the basal plane and $z=20,80,90,95$, and $180 \mathrm{~nm}$. Furthermore, a signal intensity profile for $\mathrm{SO}_{4} \mathrm{H}^{-}$in an $X Z$ slice was reported.

As already previously observed, also here the electrolyte covers up the whole basal plane. Interestingly, at $z=20 \mathrm{~nm}$, $\mathrm{SO}_{4} \mathrm{H}^{-}$ions can be found as isolated circular spots with a larger but quite constant diameter of about $20 \mu \mathrm{m}$; these circle-shaped distributions fade in and out moving towards the buried layers. In this sample, between 80 and $95 \mathrm{~nm}$, such circular spots seem to be more concentrated and distributed all over a few tens of HOPG basal planes. In view of shine a light on the evolution of such circular spots, AFM was employed to characterize the sample morphology.

In Fig. 8, the AFM image is reported. Blisters are clearly visible in the topographic image but, when the $\mathrm{SO}_{4} \mathrm{H}^{-}$chemical map is superimposed (light blue dots) there is not a perfect superposition between blisters and the investigated chemical compound (blisters do not show any enhancement of chemical species).

Furthermore, circular defects (white dashed line) are sometimes observed where ions are detected by the ToF-SIMS. On the other hand, and considering their sizes, the circular spots recall the projected area of possible blisters. These considerations suggest a correlation between the circular spots and the blisters evolution (Fig. S3 in the ESM). We thus speculate that, despite the diffusion inside the graphite bulk of solvated ions, the latter can gather in some areas (e.g., steps, defects, etc.) without any preferential directions (circular shape).

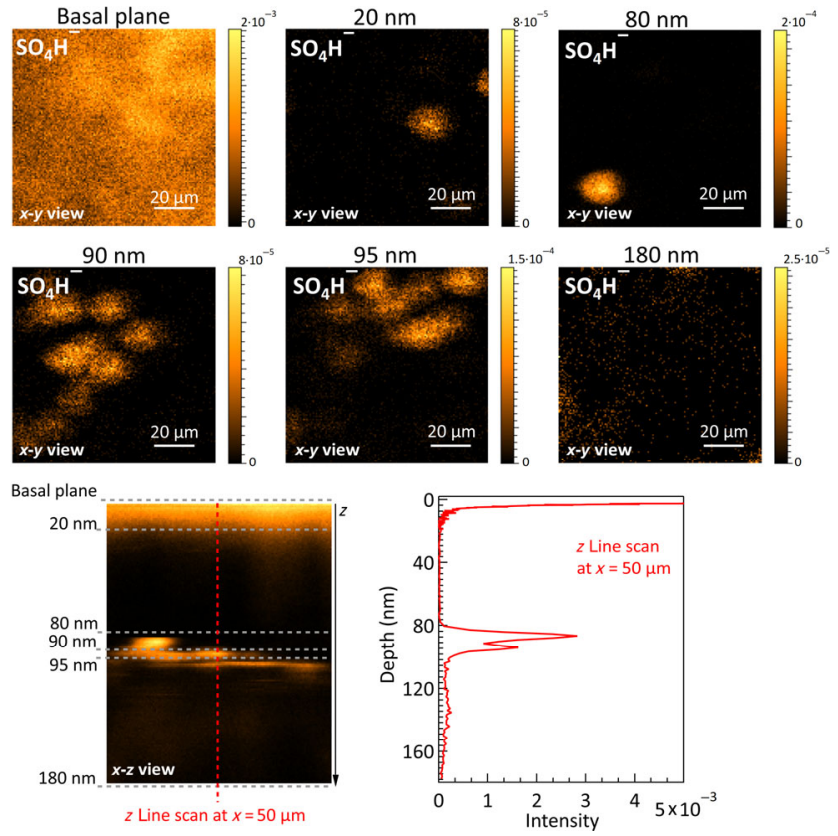

Figure $7 X Y(100 \mu \mathrm{m} \times 100 \mu \mathrm{m})$ and $X Z(z=0-180 \mathrm{~nm})$ slices reconstruction of $\mathrm{SO}_{4} \mathrm{H}^{-}$ion signal from ToF-SIMS depth profile experiments on HOPG intercalated in $\mathrm{H}_{2} \mathrm{SO}_{4}$ solution. At different depth values $(z=0,20,80,90$, 95 , and $180 \mathrm{~nm}$ ), the $X Y$ (in-plane) $\mathrm{SO}_{4} \mathrm{H}^{-}$ion intensity distribution and the corresponding $X Z$ (vertical cross-section) distribution are shown. In the bottom right panel, the line scan acquired on the $X Z$ slice (dotted red line) is plotted as depth versus intensity, in order to highlight the dimensional scale of the $\mathrm{SO}_{4} \mathrm{H}^{-}$ion segregation within the HOPG crystal.
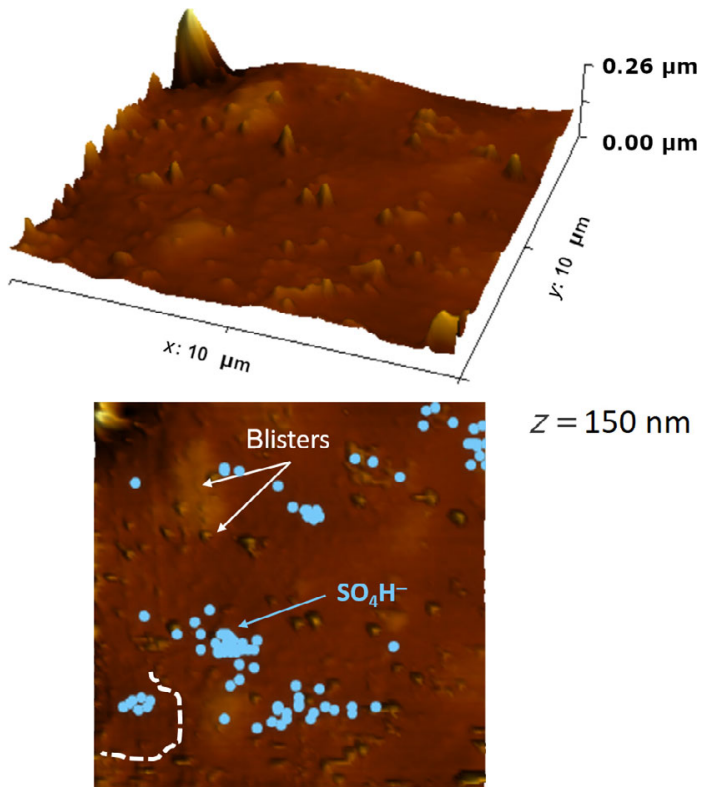

Figure 8 AFM images $(10 \mu \mathrm{m} \times 10 \mu \mathrm{m})$ of intercalated HOPG in $\mathrm{H}_{2} \mathrm{SO}_{4}$ solution, acquired on the surface sample after ToF-SIMS depth profile measurements $(z=150 \mathrm{~nm}$ ). 3D (top panel) and 2D (bottom panel) AFM topography is shown. The light blue dots on the $2 \mathrm{D}$ map represent the $\mathrm{SO}_{4} \mathrm{H}^{-}$ion signal distribution on the corresponding ToF-SIMS chemical map for this sample (data not reported).

Here, when the oxygen evolution potential is reached, graphite oxidation is enhanced and a blister can evolve. Consequently, part of the circular spots observed in the ToF-SIMS maps can be interpreted as the fingerprint of a blister that has been removed during the stratigraphic analysis. Such an interpretation is summarized as a scheme in Fig. 9. In the first steps of the electrochemical oxidative process of the graphite crystal, solvated anions are forced to intercalate in the first nanometers taking 

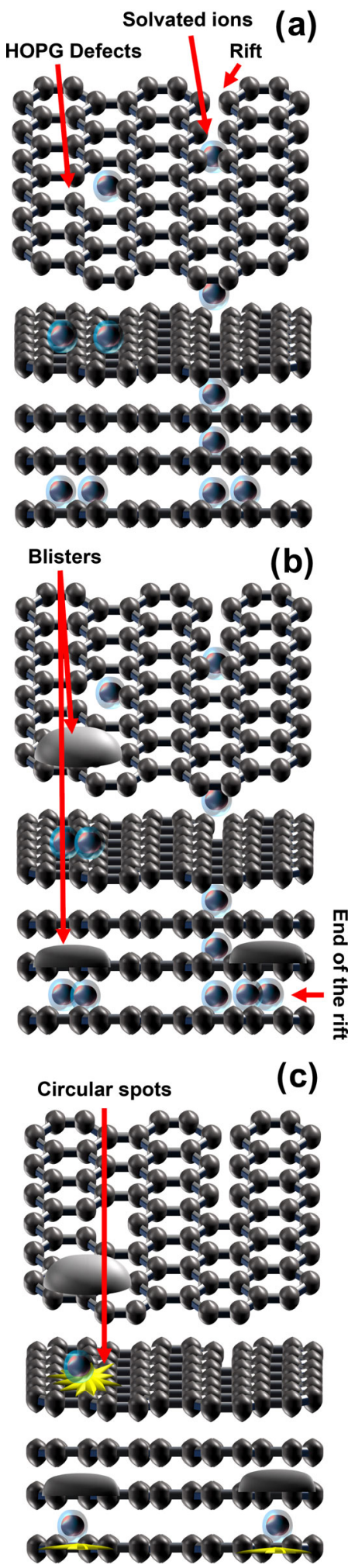

Figure 9 Definitive scheme that summarizes the interpretation of the collected results. (a) During the initial potential scans, when oxidative wave fine structures appear, solvated anions are intercalated in the first nanometers through HOPG crystal defects and grain boundaries. The presence of rifts in the graphite crystal can drive the intercalation of the solvated electrolytes even at hundreds of nanometers. (b) HOPG oxidation reactions are evidenced by the presence of acid residuals in defective areas represented as circular spots. Electrochemical graphite oxide (EGO) formation, gas evolution, and blister formation can occur at the crystal subsurface as well as in the buried layers. (c) The complete deintercalation of the electrolyte ions should occur in the reduction process during the cathodic scan. However, residuals of electrolyte are still present and located in correspondence of circular spots in the first layers and in the bulk of the graphitic electrode. advantage of the presence on the very top surface of step edges, structural defects, and grain boundaries, as reported in the theoretical model. Additionally, rifts and other longitudinal structural defects can represent an easy way for the electrolytes to directly reach the bulk of the crystal at hundreds of nanometers. There, as well as in the first graphite layers, GICs give rise to oxidative reactions with water or carbon (Fig. 9(a)). After that, the electrochemical oxidation of graphite leads to the subsequent evolution of gaseous species and formation of blister-like structures, even at great depth (Fig. 9(b)). Finally, when the reduction current wave is observed in the voltammetry, acid molecules should be completely deintercalated. However, ToF-SIMS chemical maps revealed that residuals of the electrolyte can be still detected at different depths with a characteristic circular distribution.

\section{Conclusions}

We have directly proved that the electrochemical intercalation of inorganic acids in HOPG is a process not limited to the uppermost layers of the crystal, but it concerns the first hundreds of nanometers. This was hypothesized in the past by Murray and coworkers and it actually represents the theoretical model adopted to describe the electrochemical intercalation of acid molecules in graphitic electrodes. However, this was never directly observed. The picture of the electrochemical intercalation can be now represented as a mechanism where the electrolyte molecules are forced to travel along the crystal for hundreds of nanometers. At the same time, the oxidation products are not located only at the crystal surface but can move inside the crystal. It also emerged that a crucial role is played by the large crystal defects. They can be assimilated to preferential lanes for the moving of the electrolyte molecules and represent a sort of collection point where diffusing molecules can be trapped.

\section{Acknowledgements}

The TOF-SIMS/AFM instrument was financially supported by the HERCULES foundation (now FWO). Part of this project has received funding from the European Union's Horizon 2020 research and innovation program under Grant Agreement (No. 688225) (Metro4-3D). This work has been partially funded by the National Institute for Nuclear Physics in the framework of the CSN5 Call Project FIRE (Flexible Ionizing Organic Radiation Detectors). LASR3 Surface Analysis Laboratory Roma Tre gratefully acknowledges financial support from "Fondazione Roma" (No. 5229441F37). The sample preparation has been performed in the Solid-Liquid Interface and Nanomicroscopy (SoLINano) lab that is an inter-Departmental facility of the Politecnico di Milano. We want to thank Sergio Augusto Barcelo Lins for the artwork in the graphical abstract.

Electronic Supplementary Material: Supplementary material (schematic representation of the correlative ToF-SIMS/AFM system, depth profile of a pristine HOPG sample: secondary ion intensities of $\mathrm{C}_{6}^{-}$at $m / z 72, \mathrm{O}_{2}^{-}$at $m / z 31.99, \mathrm{Cl}^{-}$at $\mathrm{m} / \mathrm{z}$ 34.96, $\mathrm{S}^{-}$at $\mathrm{m} / z$ 31.97, and $\mathrm{CO}_{2} \mathrm{H}^{-}$at $m / z 44.99$ plotted versus depth, and in-situ topography and phase-contrast images of the HOPG electrode immersed in sulfuric acid electrolyte) is available in the online version of this article at https://doi.org/ 10.1007/s12274-021-3614-6.

Funding note: Open Access funding provided by Università degli Studi Roma Tre.

Open Access This article is licensed under a Creative Commons 
Attribution 4.0 International License, which permits use, sharing, adaptation, distribution and reproduction in any medium or format, as long as you give appropriate credit to the original author(s) and the source, provide a link to the Creative Commons licence, and indicate if changes were made.

The images or other third party material in this article are included in the article's Creative Commons licence, unless indicated otherwise in a credit line to the material. If material is not included in the article's Creative Commons licence and your intended use is not permitted by statutory regulation or exceeds the permitted use, you will need to obtain permission directly from the copyright holder.

To view a copy of this licence, visit http://creativecommons.org/licenses/by/4.0/.

\section{References}

[1] Shih, C. J.; Vijayaraghavan, A.; Krishnan, R.; Sharma, R.; Han, J. H.; Ham, M. H.; Jin Z.; Lin S. C.; Paulus G. L. C.; Reuel N. F. et al. Bi- and trilayer graphene solutions. Nat. Nanotechnol. 2011, 6, 439-445.

[2] Kajiyama, S.; Szabova, L.; Sodeyama, K.; Iinuma, H.; Morita, R.; Gotoh, K.; Tateyama Y.; Okubo M.; Yamada A. Sodium-ion intercalation mechanism in MXene nanosheets. ACS Nano 2016, 10, 3334-3341.

[3] Xu, J. T.; Dou, Y. H.; Wei, Z. X.; Ma, J. M.; Deng, Y. H.; Li, Y. T.; Liu, H. K.; Dou S. X. Recent progress in graphite intercalation compounds for rechargeable metal $(\mathrm{Li}, \mathrm{Na}, \mathrm{K}, \mathrm{Al})$-ion batteries. Adv. Sci. 2017, 4, 1700146.

[4] Nicolosi, V.; Chhowalla, M.; Kanatzidis, M. G.; Strano, M. S.; Coleman, J. N. Liquid exfoliation of layered materials. Science 2013, 340, 1226419.

[5] Amiri, A.; Naraghi, M.; Ahmadi, G.; Soleymaniha, M.; Shanbedi, M. A review on liquid-phase exfoliation for scalable production of pure graphene, wrinkled, crumpled and functionalized graphene and challenges. FlatChem 2018, 8, 40-71.

[6] Liu, W.; Tanna, V. A.; Yavitt, B. M.; Dimitrakopoulos, C.; Winter, H. $\mathrm{H}$. Fast production of high-quality graphene via sequential liquid exfoliation. ACS Appl. Mater. Interfaces 2015, 7, 27027-27030.

[7] Xia, Z. Y.; Pezzini, S.; Treossi, E.; Giambastiani, G.; Corticelli, F.; Morandi, V.; Zanelli A.; Bellani V.; Palermo V. The exfoliation of graphene in liquids by electrochemical, chemical, and sonicationassisted techniques: A nanoscale study. Adv. Funct. Mater. 2013, 23, 4684-4693.

[8] Liu, F.; Wang, C. J.; Sui, X.; Riaz, M. A.; Xu, M. Y.; Wei, L.; Chen Y. Synthesis of graphene materials by electrochemical exfoliation: Recent progress and future potential. Carbon Energy 2019, 1, 173-199.

[9] Chen, H. W.; Li, C.; Qu, L. T. Solution electrochemical approach to functionalized graphene: History, progress and challenges. Carbon 2018, 140, 41-56.

[10] Alsmeyer, D. C.; McCreery, R. L. In situ Raman monitoring of electrochemical graphite intercalation and lattice damage in mild aqueous acids. Anal. Chem. 1992, 64, 1528-1533.

[11] Beck, F.; Krohn, H.; Zimmer, E. Corrosion of graphite intercalation compounds. Electrochim. Acta 1986, 31, 371-376.

[12] Alliata, D.; Häring, P.; Haas, O.; Kötz, R.; Siegenthaler, H. Anion intercalation into highly oriented pyrolytic graphite studied by electrochemical atomic force microscopy. Electrochem. Commun. 1999, 1, 5-9.

[13] Rodríguez-Pérez, I. A.; Ji, X. L. Anion hosting cathodes in dual-ion batteries. ACS Energy Lett. 2017, 2, 1762-1770.

[14] Zhou, W. C.; Sit, P. H. L. First-principles understanding of the staging properties of the graphite intercalation compounds towards dual-ion battery applications. ACS Omega 2020, 5, 18289-18300.

[15] de Boer, T.; Lapping, J. G.; Read, J. A.; Fister, T. T.; Balasubramanian, M.; Cabana, J.; Moewes, A. Direct evidence of charge transfer upon anion intercalation in graphite cathodes through new electronic states: An experimental and theoretical study of hexafluorophosphate. Chem. Mater. 2020, 32, 2036-2043.
[16] Morales, G. M.; Schifani, P.; Ellis, G.; Ballesteros, C.; Martínez, G.; Barbero, C.; Salavagione H. J. High-quality few layer graphene produced by electrochemical intercalation and microwave-assisted expansion of graphite. Carbon 2011, 49, 2809-2816.

[17] Goss, C. A.; Brumfield, J. C.; Irene, E. A.; Murray, R. W. Imaging the incipient electrochemical oxidation of highly oriented pyrolytic graphite. Anal. Chem. 1993, 65, 1378-1389.

[18] Hathcock, K. W.; Brumfield, J. C.; Goss, C. A.; Irene, E. A.; Murray, R. W. Incipient electrochemical oxidation of highly oriented pyrolytic graphite: Correlation between surface blistering and electrolyte anion intercalation. Anal. Chem. 1995, 67, 2201-2206.

[19] Didier, C.; Pang, W. K.; Guo, Z. P.; Schmid, S.; Peterson, V. K. Phase evolution and intermittent disorder in electrochemically lithiated graphite determined using in operando neutron diffraction. Chem. Mater. 2020, 32, 2518-2531.

[20] Yivlialin, R.; Bussetti, G.; Brambilla, L.; Castiglioni, C.; Tommasini, M.; Duò, L.; Passoni M.; Ghidelli M.; Casari C. S.; Bassi A. L. Microscopic analysis of the different perchlorate anions intercalation stages of graphite. J. Phys. Chem. C 2017, 121, 14246-14253.

[21] Yivlialin, R.; Bussetti, G.; Magagnin, L.; Ciccacci, F.; Duò, L. Temporal analysis of blister evolution during anion intercalation in graphite. Phys. Chem. Chem. Phys. 2017, 19, 13855-13859.

[22] Yivlialin, R.; Magagnin, L.; Duò, L.; Bussetti, G. Blister evolution time invariance at very low electrolyte $\mathrm{pH}: \mathrm{H}_{2} \mathrm{SO}_{4}$ /graphite system investigated by electrochemical atomic force microscopy. Electrochim. Acta 2018, 276, 352-361.

[23] Yivlialin, R.; Brambilla, L.; Bussetti, G.; Tommasini, M.; Li Bassi, A.; Casari, C. S.; Passoni M.; Ciccacci F.; Duò L.; Castiglioni C. Evolution of the graphite surface in phosphoric acid: An AFM and Raman study. Beilstein J. Nanotechnol. 2016, 7, 1878-1884.

[24] Lee, H.; Choi, J. I.; Park, J.; Jang, S. S.; Lee, S. W. Role of anions on electrochemical exfoliation of graphite into graphene in aqueous acids. Carbon 2020, 167, 816-825.

[25] Alliata, D.; Kötz, R.; Haas, O.; Siegenthaler, H. In situ AFM study of interlayer spacing during anion intercalation into HOPG in aqueous electrolyte. Langmuir 1999, 15, 8483-8489.

[26] Bussetti, G.; Yivlialin, R.; Alliata, D.; Li Bassi, A.; Castiglioni, C.; Tommasini, M.; Casari C. S.; Passoni M.; Biagioni P.; Ciccacci F. et al. Disclosing the early stages of electrochemical anion intercalation in graphite by a combined atomic force microscopy/scanning tunneling microscopy approach. J. Phys. Chem. C 2016, 120, 6088-6093.

[27] Jagadeesh, M. S.; Calloni, A.; Denti, I.; Goletti, C.; Ciccacci, F.; Duò, L.; Bussetti G. The effect of cyclic voltammetry speed on anion intercalation in HOPG. Surf. Sci. 2019, 681, 111-115.

[28] Bussetti, G.; Yivlialin, R.; Goletti, C.; Zani, M.; Duò, L. Temperature effects on the HOPG intercalation process. Condens. Matter 2019, $4,23$.

[29] Jagadeesh, M. S; Bussetti, G.; Calloni, A.; Yivlialin, R.; Brambilla, L.; Accogli, A.; Gibertini, E.; Alliata, D.; Goletti, C.; Ciccacci, F. et al. Incipient anion intercalation of highly oriented pyrolytic graphite close to the oxygen evolution potential: A combined x-ray photoemission and Raman spectroscopy study. J. Phys. Chem. C 2019, 123, 1790-1797.

[30] De Rosa, S.; Branchini, P.; Yivlialin, R.; Duò, L.; Bussetti, G.; Tortora L. Disclosing the graphite surface chemistry in acid solutions for anion intercalation. ACS Appl. Nano Mater. 2020, 3, 691-698.

[31] Sotgiu, C.; Tortora, L.; Petrucci, E. Influence of surface roughening of titanium substrate in the electrochemical activity of manganese oxide thin film electrode in anodic oxidation of dye-containing solutions. J. Appl. Electrochem. 2015, 45, 787-797.

[32] Zurlo, F.; Di Bartolomeo, E.; D'Epifanio, A.; Felice, V.; Natali Sora, I.; Tortora, L.; Licoccia S. $\mathrm{La}_{0.8} \mathrm{Sr}_{0.2} \mathrm{Fe}_{0.8} \mathrm{Cu}_{0.2} \mathrm{O}_{3-\delta}$ as "cobalt-free" cathode for $\mathrm{La}_{0.8} \mathrm{Sr}_{0.2} \mathrm{Ga}_{0.8} \mathrm{Mg}_{0.2} \mathrm{O}_{3-\delta}$ electrolyte. J. Power Sources 2014, 271, 187-194.

[33] Tortora, L.; Urbini, M.; Fabbri, A; Branchini, P.; Mariucci, L.; Rapisarda, M.; Barra M.; Chiarella F.; Cassinese A.; Di Capua F. et al. Three-dimensional characterization of OTFT on modified hydrophobic flexible polymeric substrate by low energy $\mathrm{Cs}^{+}$ion sputtering. Appl. Surf. Sci. 2018, 448, 628-635.

[34] Secchi, V.; Iucci, G.; Dettin, M.; Zamuner, A.; De Rosa, S.; Tortora, L.; Battocchio C. Cysteine-modified self-assembling peptides on gold: The role of the head and tail. Langmuir 2019, 35, 16593-16604. 
[35] Behrisch, R.; Eckstein, W. Sputtering by Particle Bombardment: Experiments and Computer Calculations from Threshold to $\mathrm{MeV}$ Energies; Springer: Berlin, Heidelberg, 2007.

[36] Bussetti, G.; Duò, L. Anion intercalation in graphite studied by electrochemical-scanning probe microscopy: State of the art and perspectives. In Encyclopedia of Interfacial Chemistry: Surface Science and Electrochemistry. Wandelt, K., Ed.; Elsevier: Amsterdam, 2018; pp 27-37.
[37] Yivlialin, R.; Penconi, M.; Bussetti, G.; Biroli, A. O.; Finazzi, M.; Duò, L.; Bossi A. Morphological changes of porphine films on graphite by perchloric and phosphoric electrolytes: An electrochemical-AFM study. Appl. Surf. Sci. 2018, 442, 501-506.

[38] Xie, W. J.; Weng, L. T.; Ng, K. M.; Chan, C. K.; Chan, C. M. Defects of clean graphene and sputtered graphite surfaces characterized by time-of-flight secondary ion mass spectrometry and X-ray photoelectron spectroscopy. Carbon 2017, 112, 192-200. 